\title{
Imaging Features of Breast Dermatofibrosarcoma Protuberans in Various Modalities Including FDG-PET CT
}

\author{
Suk Hyun Bae, ${ }^{1}$ and Ji Young Lee ${ }^{1,{ }^{*}}$ \\ ${ }^{1}$ Department of Radiology, Inje University College of Medicine, Ilsan Paik Hospital, Goyang-si, Republic of Korea \\ "Corresponding author: Ji Young Lee, Department of Radiology, Inje University College of Medicine, Ilsan Paik Hospital, Goyang-si, Republic of Korea. Tel: +82-319107689, E-mail: \\ drleeji@paik.ac.kr
}

Received 2015 October 18; Accepted 2015 December 23.

\begin{abstract}
Dermatofibrosarcoma protuberans is an uncommon malignant mesenchymal tumor of the dermis, which most commonly appears on the body and extremities. A preoperative diagnosis of dermatofibrosarcoma protuberans is extremely difficult, because it is a very rare entity and its appearance is often similar to that of benign breast lesion. Here, we presented a case of breast dermatofibrosarcoma protuberans with associated radiological mammography, ultrasound, magnetic resonance imaging and fludeoxyglucosepositron emission tomography computerized tomography (FDG-PET CT) features. To our knowledge, our case is the first report of PET-CT findings of breast dermatofibrosarcoma protuberans. Furthermore, we reviewed characteristic radiologic features of this rare entity, which are helpful for differentiating it from other primary benign breast lesions.
\end{abstract}

Keywords: Dermatofibrosarcoma Protuberans, Breast, Ultrasound, MRI, PET

\section{Introduction}

Dermatofibrosarcoma protuberans (DFSP) is a mesenchymal tumor of the dermis and subcutaneous tissues (1). It is a slow growing low- to intermediate-grade malignancy with a high propensity for local recurrence, especially if negative resection margins are not achieved. However, metastases are rare (2).

DFSP most commonly involves the trunk, followed by extremities, head and neck. Because occurrence in the breast is very rare, DFSP is often misdiagnosed as a benign breast tumor, leads to treatment delay. Only a few single case reports with imaging findings have been published (2). We reported a patient with DFSP of breast together with characteristic imaging features of various radiologic modalities including mammography, ultrasound, MRI and fludeoxyglucose-positron emission tomography computerized tomography (FDG-PET CT), which are necessary for an accurate diagnosis and providing a differential diagnosis from other breast lesions.

\section{Case Presentation}

A 41-year-old female presented with a lump in her left breast that she had noticed it about one month previously. Physical examination revealed a firm, tender nodule at 12o'clock position of the left breast. No regional skin color changes were observed. She had no specific clinical history or family history of breast cancer.
Mammography showed heterogeneously dense breasts that would be categorized as breast imaging reporting and data system (BI-RADS) type C. It also revealed a circumscribed, oval-shaped, equal-density mass in the subcutaneous fat layer of the upper central portion of the left breast. Associated abnormal calcification or architectural distortion was not observed (Figure $1 \mathrm{~A}$ and $\mathrm{B}$ ).

Subsequent ultrasonography performed using an acoustic gel pad demonstrated a $2.4 \times 1.0 \mathrm{~cm}$, circumscribed, oval-shaped mass in the skin and subcutaneous fat layer at the 12-o'clock position of the left breast, $4 \mathrm{~cm}$ from the nipple. The mass had a heterogeneous echo pattern with a peripheral hyperechoic rim (Figure 2A) and increased vascularity on Color Doppler sonography (Figure 2B). Malignancy was suspected for this lesion and it was categorized as BI-RADS 4. Ultrasonography-guided core biopsy was performed and spindle cell neoplasm was considered.

Dynamic contrast-enhanced breast-magnetic resonance imaging (MRI) was performed using a 1.5-T system with a dedicated breast coil, which revealed a $2.4 \times 2.0 \mathrm{~cm}$ circumscribed oval mass with a wide base to the skin. The mass was observed as low signal intensity on T1-weighted images with homogeneous strong enhancement (Figure $3 \mathrm{~A}$ and $\mathrm{B}$ ). Most sections of the tumor showed rapid initial enhancement with washout kinetics (Figure 3C).

PET-CT was performed for a routine evaluation of distant metastasis. 18F-FDG-PET demonstrated faint FDG up- 


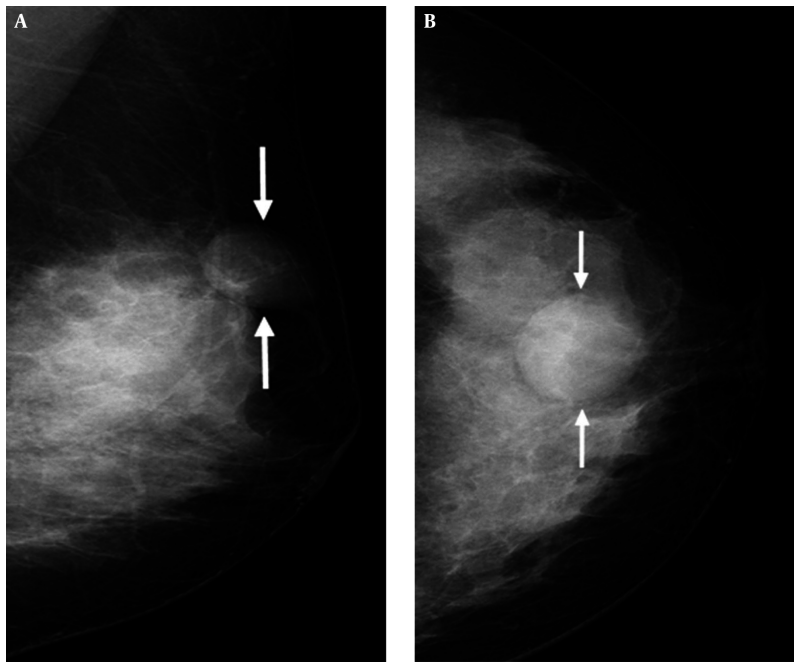

Figure 1. A 41-year-old female presented with a lump in her left breast. Mediolateral oblique view (A) and craniocaudal view (B) of mammography shows dense breast and a circumscribed, oval-shaped, equal-density mass (arrows) in the subcutaneous fat layer of the upper central portion of the left breast.
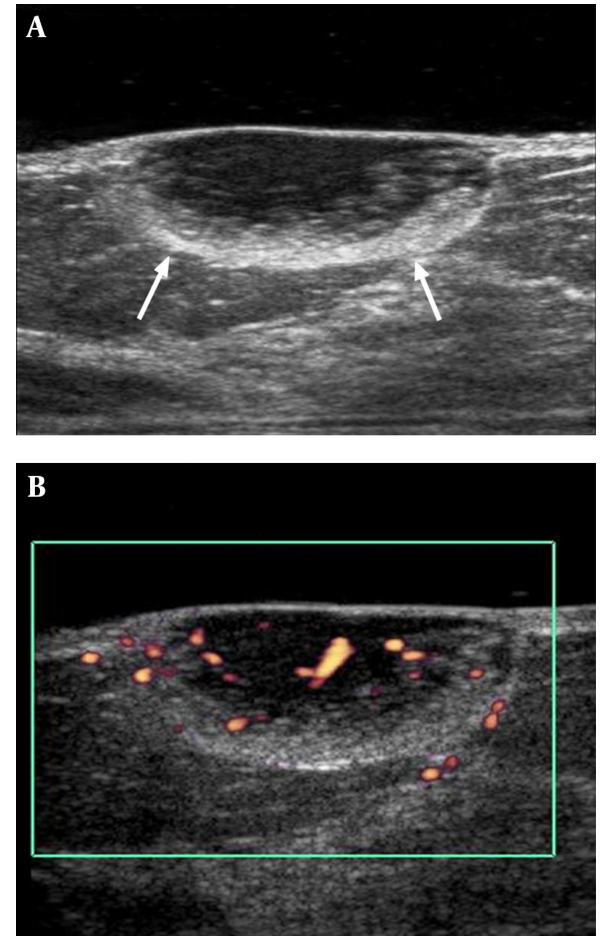

Figure 2. A, Gray scale image of ultrasonography showing a $2.4 \times 1.0 \mathrm{~cm}$ circumscribed, oval-shaped, heterogeneous echoic mass with peripheral hyperechoic rim portion (arrows) in the subcutaneous fat layer at the 12-o'clock position of the left breast, $4 \mathrm{~cm}$ from the nipple; $B$, Color Doppler image of ultrasonography showing increased vascularity in mass.

take around the mass in the left breast and standardized uptake value (SUV) was measured as 1.8 (Figure $4 \mathrm{~A}$ and B). Other soft tissue FDG uptake or evidence of distant metas- tasis was not observed.

The patient underwent local mass excision with $3 \mathrm{~cm}$ margins. Histologic examination revealed infiltration of 

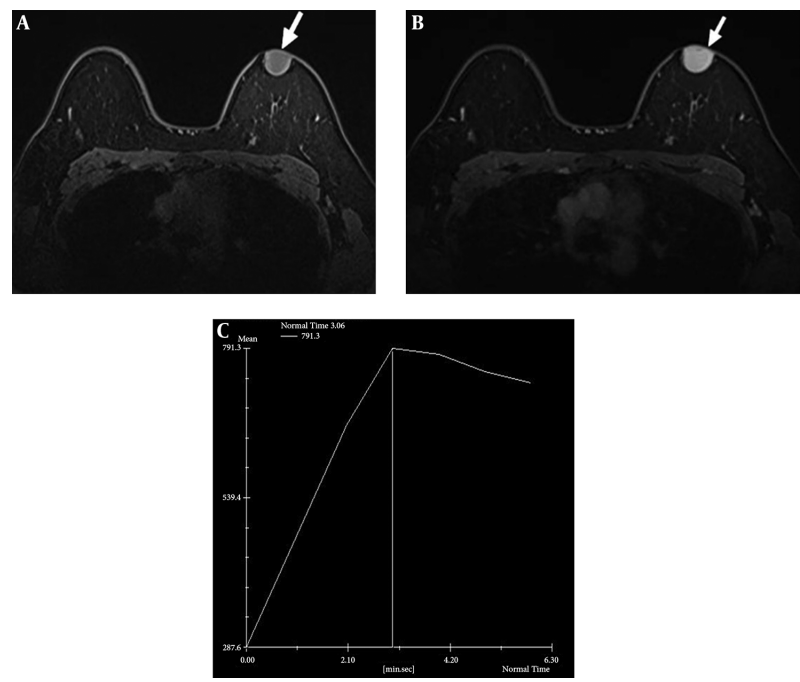

Figure 3. Axial T1-weighted magnetic resonance image shows circumscribed oval low signal intensity mass in the left breast(A, arrow) and homogeneous strong enhancement in majority of mass could be seen after contrast injection (B, arrow). Kinetic curve shows early strong enhancement with washout (C).
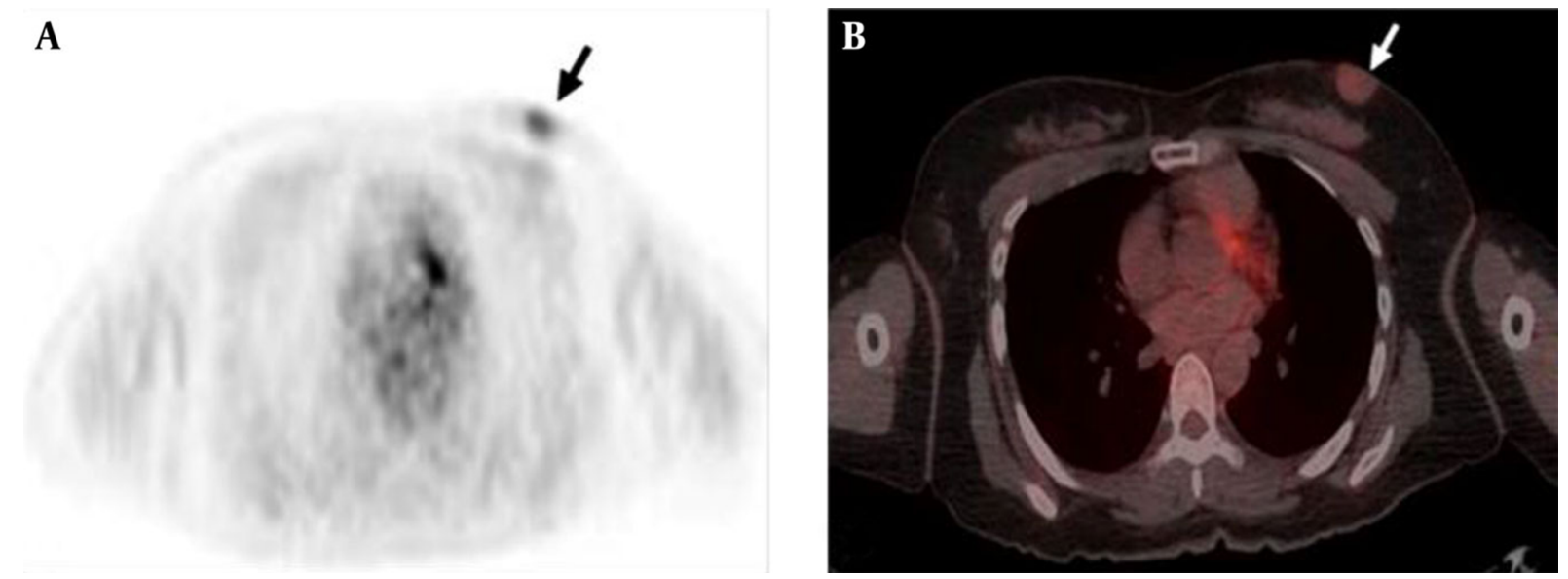

Figure 4. A, Axial view of 18F-FDG PET-CT image and B, Fusion PET-CT image showing a focal 18F-FDG uptake in the left breast with SUVmax as 1.8 (arrow).

a $2.0 \times 1.5 \times 2.0 \mathrm{~cm}$ sized tumor into the subcutaneous fat on low-power field view and storiform arrangement of short spindle cells on high-power field view. The tumor cells were diffusely positive for CD34 immunostaining(Figure 5). Therefore, histologic diagnosis was DFSP. Tumor recurrence was not observed during sonographic follow-up 4 years after surgery.

\section{Discussion}

DFSP is a rare cutaneous malignancy that arises from dermis and subcutaneous fat layer. The reported incidence is around 5 cases per 1 million persons annually and is most commonly found in adults aged 20 and 50 years (1). Although the tumor is a low-to-intermediate grade sarcoma, it can infiltrate into the surrounding deeper tissue with a propensity for local recurrence, especially after inadequate excision. While metastasis is rare, it can occur in distant sites, especially the lung (2-4). Furthermore, differentiation into higher-grade sarcomas with higher metastatic potential tends to occur in poorly treated recurrent tumors (1).

DFSP most commonly appears on the trunk (50 - 60\%), extremities (25\%) and in the head and neck (10 - 15\%). Although breast involvement is quite unusual, it has been reported in the literature with imaging features (1). 

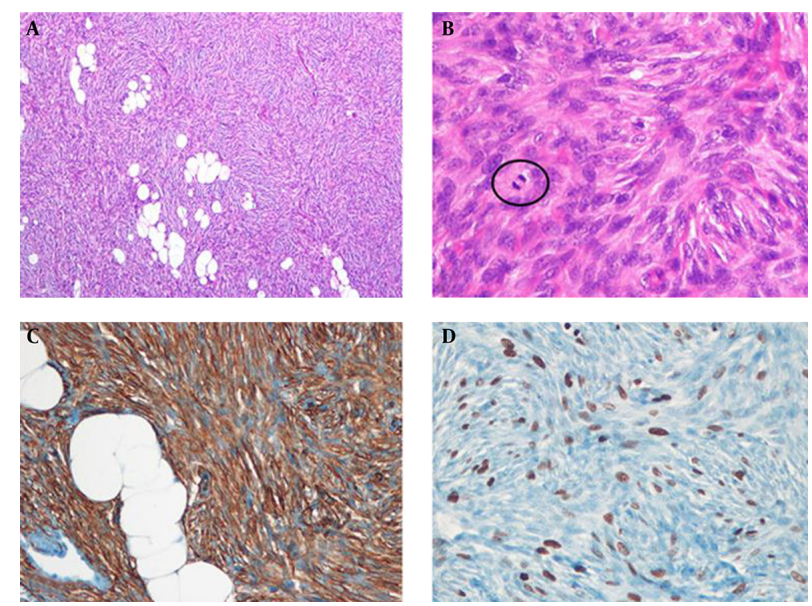

Figure 5. Histopathologic findings of the left breast mass. A, At low magnification view, the tumor is homogeneously cellular and infiltrates into subcutaneous fat (Hematoxylin and eosin (H \& E) stain, $\times 40)$; B, High power view demonstrates storiform arrangement of short spindle cells and mitotic figures (circle) $(\mathrm{H} \& \mathrm{E}$ stain, $\times 400)$; $\mathrm{C}$ Tumor cells have diffuse positive results for CD34 immunostaining $(\times 200)$; D, Ki-67 labeling index is $25 \%(\times 200)$.

The reported mammographic findings of DFSP are nonspecific, i.e. a skin-based or intramammary-located, oval, circumscribed mass without calcification that mimics a benign lesion $(1,2,5)$. These features are consistent with our case, in which a circumscribed, equal- density, oval mass without calcification was observed in superficially subcutaneous fat layer of the upper central portion of the left breast.

The characteristic ultrasonographic features of DFSP of breast were a circumscribed, hypoechoic, subcutaneous mass with increased internal blood flow in Color Doppler image $(4,6,7)$. Especially, hypervascularity of the tumor helps it to be differentiated from other benign breast lesions. Although a breast abscess or complicated epidermal inclusion cyst such as infection or rupture can appear as a hypoechoic mass with a thick echogenic periphery and increased vascular flow (8). However, our patient had no clinical symptoms associated with infection such as pain, redness, a sense of heating or fever. Another distinct ultrasonographic feature of DFSP of the breast is a peripheral hyperechoic band like rim in the subcutaneous tissue, which represents a mixture of tumor cells and fibrous tissue infiltrating the subcutaneous fat (2).

Although the signal intensity of DFSP on breast MR imaging is nonspecific, MRI is the most helpful imaging modality for evaluating the tumor extent and anatomical relationships with surrounding structures. Compared to ultrasound, MRI represents more accurately the tumor size and the boundary because of excellent contrast with surrounding tissue. MR appearance of breast DFSP is a wellcircumscribed, intermediate low-intensity tumor on both T1 and T2-weighted images with washout-type kinetics and strong enhancement (4). We also observed a low signal intensity mass on T1-weighted images with initially rapid strong enhancement with washout kinetics in our case.

Although DFSP has a high tendency for local recurrence after insufficient surgical excision, metastasis is rare. However, distant metastasis may occur at the lung, bone or regional lymph nodes after repeated local recurrence $(9,10)$. FDG-PET is a useful tool for predicting tumor recurrence and distant metastasis, detecting multicentric tumor origin and providing relevant anatomical information (11). A few reports have described PET-CT findings of DFSP including mild uptake in the skin of the trunk and head or more marked uptake at metastatic sites in the lung, retroperitoneum or muscles $(12,13)$.

Folpe et al. (14) reported a highly significant relationship between the highest PET SUV values and cellularity and proliferative indices in sarcomas. In the present case, the tumor showed mild FDG uptake with a SUVmax of 1.8. Although a correlation between FDG uptake and histologic grade in DFSP has not been reported in the literature, we assume that low SUV of the DFSP of the breast was attributable to low cellularity and proliferative indices. To our knowledge, this is the first case showing 18F-FDG PET findings of breast DFSP.

In conclusion, breast DFSP is a rare sarcoma that may simulate a primary breast mass both clinically and radiographically. We described a case of breast DFSP and presented radiologic features that can potentially help provide a differential diagnosis between DFSP and primary benign breast lesions. Mammography, ultrasound, MRI and FDG-PET findings of a subcutaneously located, highly vascular, non-inflammatory tumor with a wide base to the 
skin might be suggestive of DFSP.

\section{Footnotes}

Authors' Contribution: Study concept and design: Dr. Lee; acquisition of data: Dr. Lee and Dr. Bae; analysis and interpretation of data: Dr. Lee and Dr. Bae; drafting of the manuscript: Dr. Lee and Dr. Bae; critical revision of the manuscript for important intellectual content: Dr. Lee; administrative, technical and material support: Dr. Lee; study supervision: Dr. Lee.

Funding/Supports: This work was supported by the Inje Research and Scholarship Foundation in 2013.

\section{References}

1. Sin FN, Wong KW. Dermatofibrosarcoma protuberans of the breast: a case report. Clin Imaging. 2011;35(5):398-400. doi: 10.1016/j.clinimag.2011.04.004. [PubMed: 21872132].

2. Lee SJ, Mahoney MC, Shaughnessy E. Dermatofibrosarcoma protuberans of the breast: imaging features and review of the literature. AJR Am J Roentgenol. 2009;193(1):W64-9. doi: 10.2214/AJR.08.2141. [PubMed: 19542385].

3. Jiang JQ, Huang Z, Wang LH, Shen SD, Lu H. Dermatofibrosarcoma protuberans of the breast: A case report. Oncol Lett. 2014;8(3):1202-4. doi: 10.3892/ol.2014.2291. [PubMed: 25120687].

4. Djilas-Ivanovic D, Prvulovic N, Bogdanovic-Stojanovic D, Vicko F, Sveljo O, Ivkovic-Kapicl T. Dermatofibrosarcoma protuberans of the breast: mammographic, ultrasound, MRI and MRS features. Arch Gynecol Obstet. 2009;280(5):827-30. doi: 10.1007/s00404-009-1004-5. [PubMed: 19252921].

5. Cottier O, Fiche M, Meuwly JY, Delaloye JF. Dermatofibrosarcoma presenting as a nodule in the breast of a 75-year-old woman: a case report. J Med Case Rep. 2011;5:503. doi: 10.1186/1752-1947-5-503. [PubMed: 21975420].
6. Shin YR, Kim JY, Sung MS, Jung JH. Sonographic findings of dermatofibrosarcoma protuberans with pathologic correlation. J Ultrasound Med. 2008;27(2):269-74. [PubMed:18204018].

7. Kau T, Lesnik G, Arnold G, Hausegger KA. Sonography of dermatofibrosarcoma protuberans of the groin. J Clin Ultrasound. 2008;36(8):520-2. doi: 10.1002/jcu.20484. [PubMed: 18431749].

8. Trop I, Dugas A, David J, El Khoury M, Boileau JF, Larouche N, et al. Breast abscesses: evidence-based algorithms for diagnosis, management, and follow-up. Radiographics. 2011;31(6):1683-99. doi: 10.1148/rg.316115521. [PubMed: 21997989].

9. Kim SM, Rha EY, Jung SN, Lim JS, Yoo G, Byeon JH. Dermatofibrosarcoma protuberans with pulmonary metastasis in the absence of local recurrence. Arch Plast Surg. 2012;39(3):265-7. doi: 10.5999/aps.2012.39.3.265. [PubMed: 22783540].

10. Al Tarakji M, Toro A, Di Carlo I, Junejo K. Unusual presentation of dermatofibrosarcoma protuberans in a male patient's breast: a case report and review of the literature. World J Surg Oncol. 2015;13:158. doi: 10.1186/s12957-015-0562-1. [PubMed: 25896773]

11. Ahn SG, Park JT, Lee HM, Lee HW, Jeon TJ, Han K, et al. Standardized uptake value of (1)(8)F-fluorodeoxyglucose positron emission tomography for prediction of tumor recurrence in breast cancer beyond tumor burden. Breast Cancer Res. 2014;16(6):502. doi: 10.1186/s13058-0140502-y. [PubMed: 25551703].

12. Al-Tamimi A, Zaheer S, Pierce CK, Osmany S, Sittampalam K. Recurrent dermatofibrosarcoma protuberans of the shoulder with rare distant abdominal metastasis detected by fluorodeoxyglucose-positron emission tomography/computed tomography (FDG-PET/CT). Sultan Qaboos Univ Med J. 2012;12(3):371-4. doi: 10.12816/0003156.

13. Basu S, Baghel NS. Recurrence of dermatofibrosarcoma protuberans in post-surgical scar detected by 18F-FDG-PET imaging. Hell J Nucl Med. 2009;12(1):68. [PubMed: 19330190].

14. Folpe AL, Lyles RH, Sprouse JT, Conrad E3, Eary JF. (F-18) fluorodeoxyglucose positron emission tomography as a predictor of pathologic grade and other prognostic variables in bone and soft tissue sarcoma. Clin Cancer Res. 2000;6(4):1279-87. [PubMed: 10778952]. 